\title{
On the Nonexistence of $k$-reptile Tetrahedra
}

\author{
Jiří Matoušek · Zuzana Safernová
}

Received: 7 June 2010 / Revised: 14 February 2011 / Accepted: 17 February 2011 /

Published online: 4 March 2011

(C) Springer Science+Business Media, LLC 2011

\begin{abstract}
A $d$-dimensional simplex $S$ is called a $k$-reptile if it can be tiled without overlaps by simplices $S_{1}, S_{2}, \ldots, S_{k}$ that are all mutually congruent, and similar to $S$. For $d=2, k$-reptile simplices (triangles) exist for many values of $k$, and they have been completely characterized by Snover, Waiveris, and Williams. On the other hand, for $d \geq 3$, only one construction of $k$-reptile simplices is known, the Hill simplices, and it provides only $k$ of the form $m^{d}, m=2,3, \ldots$.

We prove that for $d=3, k$-reptile simplices (tetrahedra) exist only for $k=m^{3}$. This partially confirms a conjecture of Hertel, asserting that the only $k$-reptile tetrahedra are the Hill tetrahedra.

Our research has been motivated by the problem of probabilistic packet marking in theoretical computer science, introduced by Adler in 2002.
\end{abstract}

Keywords Reptile $\cdot$ Tiling $\cdot$ Self-similarity $\cdot$ Geometry of a simplex

\section{Introduction}

A closed set $X \subset \mathbb{R}^{d}$ with nonempty interior is called a $k$-reptile (sometimes written " $k$ rep tile" or " $k$ rep-tile") if there are sets $X_{1}, X_{2}, \ldots, X_{k}$ with disjoint interiors and with $X=X_{1} \cup X_{2} \cup \cdots \cup X_{k}$ that are all mutually congruent, and similar to $X$. Such

Zuzana Safernová was supported by the Charles University grant GAUK 52110.

J. Matoušek ( $₫)$

Department of Applied Mathematics and Institute of Theoretical Computer Science (ITI), Charles

University, Malostranské nám. 25, 11800 Praha 1, Czech Republic

e-mail: matousek@kam.mff.cuni.cz

Z. Safernová

Department of Applied Mathematics, Charles University, Malostranské nám. 25, 11800 Praha 1,

Czech Republic

e-mail: zuzka@kam.mff.cuni.cz 
Fig. 1 A three-dimensional Hill simplex as an 8-reptile
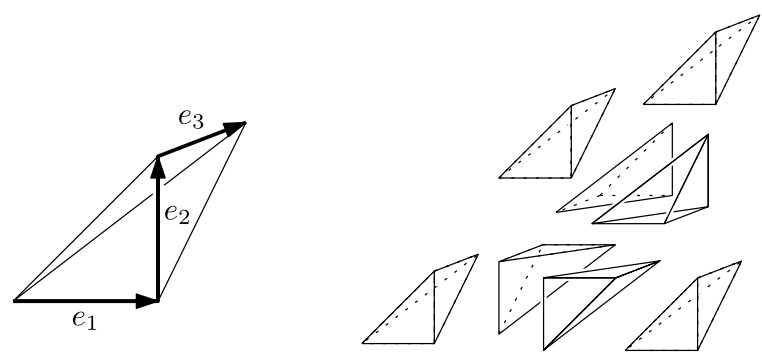

sets have been studied in connection with fractals and also with crystallography and tilings of $\mathbb{R}^{d}$; see, for example, $[3,8,9,16,21]$.

Here we consider the following question: For what $k$ and $d$ there exist $d$-dimensional simplices that are $k$-reptiles? This investigation was motivated by a paper of Adler [1] on probabilistic marking of Internet packets. The connection and the quite interesting questions arising there are discussed in [2] or, in a more concise form, in [15]. From this point of view, it would be interesting to find $d$-dimensional $k$-reptile simplices with $k$ as small as possible.

The simplest $k$-reptile simplex, for $d=k=2$, is the isosceles right triangle (with angles 45,45 , and 90 degrees). There are several possible types of $k$-reptile triangles, and they have been completely classified by Snover et al. [21]. In particular, $k$-reptile triangles exist for all $k$ of the form $a^{2}+b^{2}$ or $3 a^{2}$ for arbitrary integers $a, b$.

In contrast, for $d \geq 3$, reptile simplices seem to be much more rare. The only known construction, at least as far as we could find, of higher-dimensional $k$-reptile simplices has $k=m^{d}$ and is known as the Hill simplex (or Hadwiger-Hill simplex) [12]. A $d$-dimensional Hill simplex is the convex hull of vectors $0, b_{1}$, $b_{1}+b_{2}, \ldots, b_{1}+\cdots+b_{d}$, where $b_{1}, b_{2}, \ldots, b_{d}$ are vectors of equal length such that the angle between every two of them is the same and lies in the interval $\left(0, \frac{2 \pi}{3}\right)$. Figure 1 shows the decomposition of a three-dimensional Hill simplex, with $\left(b_{1}, b_{2}, b_{3}\right)=\left(e_{1}, e_{2}, e_{3}\right)$ the standard orthonormal basis, into 8 congruent pieces similar to it.

Concerning nonexistence of $k$-reptile simplices in dimension $d \geq 3$, Hertel [11] proved that a three-dimensional simplex is an $\mathrm{m}^{3}$-reptile using a "standard" way of dissection (which we will not define here) if and only if it is a Hill simplex. He conjectured that Hill simplices are the only three-dimensional reptile simplices. The first author [15] showed that there are no 2-reptile simplices of dimension 3 or larger.

We prove the following result.

Theorem 1.1 For $d=3$, $k$-reptile simplices (tetrahedra) exist only for $k$ of the form $m^{3}, m=2,3, \ldots$, and in particular, no $k$-reptile tetrahedra exist with $k \leq 7$.

The case $k=2$ is an (easy) special case of the result of [15] mentioned above. The starting point of the proof in [15] is observing that there is essentially only one way of partitioning a simplex into two simplices (in any dimension).

The case $k=3$, i.e., nonexistence of 3-reptile tetrahedra, was also established earlier, by the second author in her Bc. thesis [18]. The proof again discusses all 
geometric possibilities of how a tetrahedron can be partitioned into three tetrahedra. There are five cases to consider, and it seems clear that for larger $k$, keeping track of all geometric partitions quickly becomes unmanageable.

Our proof of Theorem 1.1 uses other tools, and among others, results related to Hilbert's third problem on equidecomposability, or scissors congruence, of polyhedra (a recent and very nice discussion of this area can be found in [17], and a more classical source is [4]). The proof ultimately relies on a case analysis, but with only few cases to consider, and while some of the steps are clearly specific for dimension 3 , we believe that some of the ideas may be useful for attacking higher-dimensional cases as well.

It is well known, and easy to see, that whenever $S$ is a $d$-dimensional $k$-reptile simplex, then all of $\mathbb{R}^{d}$ can be tiled by congruent copies of $S$ (indeed, using the tiling of $S$ by its smaller copies $S_{1}, \ldots, S_{k}$ as a pattern, one can inductively tile larger and larger similar copies of $S$ ). The question of characterizing the tetrahedra that tile $\mathbb{R}^{3}$ is an open and apparently rather difficult question. Several papers have been devoted to it, e.g., [5, 10, 19, 20], but they contain mainly existence results (i.e., constructions of tilings), and mostly they do not seem to be directly relevant to the $k$-reptile question.

Open problems Concerning the existence of $k$-reptile simplices in dimensions $d \geq 4$, we dare to state the following conjecture:

Conjecture 1.2 If $d \geq 3$ and a d-dimensional $k$-reptile simplex exists, then $k=m^{d}$ for some natural number $m \geq 2$.

For $d=3$, it remains to find out whether all $m^{3}$-reptile simplices are indeed Hill simplices, as conjectured by Hertel, or whether some other types may exist as well.

Remark Jan Kynčl and the second author have recently proved that a four-dimensional $k$-reptile simplex can exist only for $k=m^{2}$, where $m \geq 2$ [14]. It remains an open question whether there exists a four-dimensional $\mathrm{m}^{2}$-reptile simplex for nonsquare $m$. They have also simplified the proof of Theorem 1.1.

\section{Preliminaries}

Scissors Congruence of Polyhedra Two convex polytopes in $\mathbb{R}^{d}$ are called scissorscongruent if the first can be cut into finitely many convex polytopes that can be reassembled to yield the second. We recall some results on scissors congruence; proofs and references can be found, e.g., in [17]. A convex polytope $P$ is called rectifiable if it is scissors-congruent to a cube.

A dihedral angle of a convex polytope in $\mathbb{R}^{3}$ is the internal angle of two facets of $P$ that meet in an edge. (For example, a tetrahedron has 6 dihedral angles.) Bricard's condition asserts that if $P$ is rectifiable, then the number $\pi$ can be written as a linear combination of all the dihedral angles of $P$ with strictly positive rational coefficients.

A convex polytope $P$ is called self-similar if it is scissors-congruent to a disjoint union of two or more polytopes, each of them similar to $P$. In particular, a $k$-reptile 
simplex is self-similar. According to Sydler's criterion, a convex polytope $P \subset \mathbb{R}^{3}$ is rectifiable if and only if it is self-similar. Thus, we have the following consequence.

Fact 2.1 Let $S$ be a $k$-reptile tetrahedron for some $k$, and let $\alpha_{1}, \ldots, \alpha_{6}$ be its dihedral angles (not necessarily all distinct). Then there are strictly positive rational numbers $q_{1}, \ldots, q_{6}$ such that

$$
\sum_{i=1}^{6} q_{i} \alpha_{i}=\pi .
$$

Existence of Simplices with Given Dihedral Angles In the forthcoming proof of Theorem 1.1, we will need to exclude the existence of tetrahedra with specified dihedral angles. We can use the following elegant condition due to Fiedler, which we state for an arbitrary dimension $d$.

For a $d$-dimensional simplex $S$, let us number the facets as $F_{1}, \ldots, F_{d+1}$, let $v_{i}$ be the vertex opposite to $F_{i}$, and let $\varphi_{i j}$ be the dihedral angle of $F_{i}$ and $F_{j}\left(\operatorname{so} \varphi_{i j}=\varphi_{j i}\right.$ ). Moreover, for technical convenience, we define $\varphi_{i i}=\pi$ for all $i$.

Theorem 2.2 Let the $\varphi_{i j}, i, j=1,2, \ldots, d+1$, be as above for some d-dimensional simplex $S$, and let $A$ be the $(d+1) \times(d+1)$ matrix with $a_{i j}:=\cos \varphi_{i j}$ for all $i, j$. Then $A$ is negative semidefinite of rank $d$, and the (one-dimensional) kernel of $A$ is generated by a vector $z \in \mathbb{R}^{d+1}$ with all components strictly positive.

This result is an immediate consequence of [6, Theorem 6]. Since we have not found any published proof in English, we include one proof in the spirit of Fiedler's recent lecture notes [7].

Proof Let $u_{i}$ be the unit normal of $F_{i}$ pointing inside $S$. Then $a_{i j}=\cos \varphi_{i j}=$ $-\left\langle u_{i}, u_{j}\right\rangle$, where $\langle\cdot, \cdot\rangle$ denotes the scalar product, and so $-A$ is the Gram matrix of the $u_{i}$. Thus, $A$ is negative semidefinite of rank $d$.

After translation, we may assume that $v_{d+1}=0$. Then $v_{j}$ is contained in $F_{i}$ for $i \neq j$, while $v_{i}$ lies on the side of $F_{i}$ where $u_{i}$ points to, $1 \leq i, j \leq d$. Thus, $\left\langle u_{i}, v_{j}\right\rangle=0$ for $i \neq j$ and $\left\langle u_{i}, v_{i}\right\rangle>0$, again for $1 \leq i, j \leq d$. Similarly, considering the facet $F_{d+1}$, we get $\left\langle u_{d+1}, v_{i}\right\rangle<0$ and $\left\langle u_{d+1}, v_{i}-v_{j}\right\rangle=0$ for all $i, j=$ $1,2, \ldots, d$.

Now we define the vector $z$ generating the kernel of $A$ : For $i=1,2, \ldots, d$, we set $z_{i}:=\left\langle u_{i}, v_{i}\right\rangle^{-1}$, and we put $z_{d+1}:=\|w\|$, where $w=-\sum_{i=1}^{d} z_{i} u_{i}$, and $\|\cdot\|$ denotes the Euclidean norm. Since $z_{1}, \ldots, z_{d}>0$, we have $w \neq 0$ by the linear independence of $u_{1}, \ldots, u_{d}$, and thus $z=\left(z_{1}, \ldots, z_{d+1}\right)$ has all components strictly positive.

It remains to show that $A z=0$; in other words, that $\sum_{i=1}^{d+1} z_{i}\left\langle u_{i}, u_{j}\right\rangle=0$ for all $j$. To this end, it suffices to check that $\sum_{i=1}^{d+1} z_{i} u_{i}=0$.

By definition, $\sum_{i=1}^{d} z_{i} u_{i}=-w$, so we need to show that $z_{d+1} u_{d+1}=w$. Since $z_{d+1}=\|w\|$, we should verify that $w$ is parallel to $u_{d+1}$ and has the same orientation.

We have $\left\langle w, v_{j}\right\rangle=-\sum_{i=1}^{d} z_{i}\left\langle u_{i}, v_{j}\right\rangle=-z_{j}\left\langle u_{j}, v_{j}\right\rangle=-1$. Thus, $\left\langle w, v_{j}-v_{k}\right\rangle=$ 0 for all $j, k=1,2, \ldots, d$, and so $w$ is indeed parallel to $u_{d+1}$, and $\left\langle w, v_{1}\right\rangle<0$, and so $u_{d+1}$ and $w$ have the same orientation. 
We will use the following consequence of Theorem 2.2 several times.

Corollary 2.3 If $A$ and the $\varphi_{i j}$ are as in Theorem 2.2, then the row space of A cannot contain a nonzero vector with all entries nonnegative (or all entries nonpositive).

We will also need the following fact (see, e.g., Fiedler [6, Theorem 8]; here we do not reproduce a proof, since in the single instance where we use the fact, one can easily give an alternative argument).

Lemma 2.4 A simplex is determined by its dihedral angles, uniquely up to similarity.

Here is another useful fact concerning the dihedral angles of a tetrahedron.

Observation 2.5 The three dihedral angles adjacent to a vertex of a tetrahedron have the sum greater than $\pi$.

Sketch of proof This follows from the fact that the sum of the angles of a spherical triangle exceeds $\pi$.

On Rational Dihedral Angles Let us call an angle $\alpha$ rational if it is a rational multiple of $\pi$ or, equivalently, if its value in degrees is rational. We will need the following result of Jahnel [13] concerning the values of the cosine for rational angles.

Theorem 2.6 Let $\alpha=\frac{2 \pi m}{n}$ be a rational angle, where $m, n$ are coprime integers (and $n \neq 0)$. Then

(i) $\cos \alpha$ is a rational number if and only if $\varphi(n) \leq 2$, where $\varphi(\cdot)$ denotes the Euler totient function, and

(ii) $\cos \alpha$ is an algebraic number of degree $d \geq 2$ if and only if $\varphi(n)=2 d$.

Jahnel's proof is short, and we sketch it: Since $t:=\cos \alpha=\left(\xi+\xi^{-1}\right) / 2$, where $\xi=e^{2 \pi i m / n}$ is a primitive $n$th root of unity, $\xi$ is a root of the quadratic equation $x^{2}-2 t x+1=0$, and hence, $[\mathbb{Q}(\xi): \mathbb{Q}(t)] \leq 2$. For $n \geq 3$, we have $[\mathbb{Q}(\xi): \mathbb{Q}(t)]=2$ since $\xi$ is not real, and using $[\mathbb{Q}(\xi): \mathbb{Q}]=\varphi(n)$, we get $[\mathbb{Q}(t): \mathbb{Q}]=\frac{\varphi(n)}{2}$.

\section{The Proof}

Here we prove Theorem 1.1. For contradiction, we assume, from now on, that $S$ is a $k$-reptile tetrahedron, where $k$ is not a third power of a natural number.

Let $S_{1}, \ldots, S_{k}$ be the mutually congruent simplices similar to $S$ that tile $S$, as in the definition of a $k$-reptile. Then each $S_{i}$ has volume $k$-times smaller than $S$, and thus $S_{i}$ is scaled by the ratio $\rho:=k^{-1 / 3}$ compared to $S$.

As is well known, $\rho$ is irrational. We will need a stronger property: $\rho$ has degree 3 over $\mathbb{Q}$, and thus it is not the root of a quadratic polynomial with integer coefficients. (Indeed, if the polynomial $k x^{3}-1$ were reducible over the rationals, then it would 
have a linear factor and thus a rational root, which is not the case, and therefore, it is irreducible.)

Let $D \subseteq \mathbb{R}$ denote the set of the dihedral angles of $S$. We have $|D| \leq 6$, since $S$ has 6 edges, but it may happen that $|D|<6$, since the same dihedral angle may appear at several edges.

Let us say that a dihedral angle $\alpha \in D$ is indivisible in $D$ if it cannot be written as a linear combination of other elements of $D$ with nonnegative integer coefficients. (In particular, the smallest dihedral angle $\alpha_{\min } \in D$ is indivisible in $D$.)

Here is a key lemma in the proof, which allows us to reduce the possible shapes of the considered tetrahedron to a manageable number of cases.

Lemma 3.1 If $\alpha \in D$ is indivisible in $D$, then the edges of $S$ with dihedral angle $\alpha$ have at least three different lengths (and in particular, there are at least three such edges).

Proof Let $e$ be an edge of $S$ with dihedral angle $\alpha$. Every point of $e$ belongs to some edge of some of the smaller simplices $S_{i}$. Since $\alpha$ is indivisible in $D$, we get that $e$ is tiled by edges of the $S_{i}$, and each of the edges in this tiling also has dihedral angle $\alpha$ in the appropriate $S_{i}$.

For contradiction, let us assume that the lengths of all the edges of $S$ with dihedral angle $\alpha$ belong to the set $\left\{x_{1}, x_{2}\right\}$, where $x_{1}, x_{2}$ are some strictly positive reals (we also admit $x_{1}=x_{2}$ ). Then, by the above, an edge of length $x_{1}$ in $S$ is tiled by edges with lengths $\rho x_{1}$ and $\rho x_{2}$, and similarly for $x_{2}$. Thus, we get that there are nonnegative integers $n_{i j}, i, j=1,2$, such that

$$
\begin{aligned}
& n_{11} \rho x_{1}+n_{12} \rho x_{2}=x_{1}, \\
& n_{21} \rho x_{1}+n_{22} \rho x_{2}=x_{2} .
\end{aligned}
$$

If we now regard $x_{1}, x_{2}$ as unknowns, then (1) is a homogeneous system of two linear equations in two unknowns. Since we assume that there is a nonzero solution, the two equations must be linearly dependent, and thus the determinant of this system vanishes. This leads to

$$
\left(n_{11} n_{22}-n_{12} n_{21}\right) \rho^{2}-\left(n_{11}+n_{22}\right) \rho+1=0 .
$$

Thus, $\rho$ should satisfy a quadratic equation with integer coefficients, but, as was mentioned earlier, it does not. This is a contradiction proving the lemma.

Here is another condition on the dihedral angles, resembling Fact 2.1 but much simpler.

Lemma 3.2 There are nonnegative integers $i_{\alpha}, \alpha \in D$, such that $\sum_{\alpha \in D} i_{\alpha} \alpha=\pi$.

Proof Consider a facet $F$ of $S$ of the largest area. Then $F$ cannot be covered by a facet of any $S_{i}$, and thus there is an edge of some $S_{i}$ going through the relative interior of $F$. We choose a point $x$ on this edge that is not a vertex of any $S_{i}$. The lemma follows by considering the dihedral angles of those edges of the $S_{i}$ that contain $x$. 
Fig. 2 Two possible configurations of two dihedral angles: the path configuration (left), and the triangle-tripod configuration (right). Black edges correspond to one of the dihedral angles, and gray edges to the other
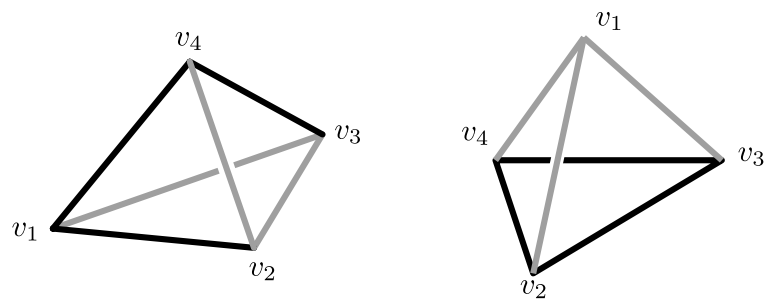

The next lemma describes two possible structures of $D$.

Lemma 3.3 One of the following two possibilities occur:

(i) All the dihedral angles of $S$ are integer multiples of the minimal dihedral angle $\alpha_{\min }$, which has the form $\frac{\pi}{n}$ for an integer $n \geq 3$.

(ii) There are exactly two distinct dihedral angles $\beta_{1}$ and $\beta_{2}$, each of them occurring three times in $S$.

Proof We select elements $\beta_{1}<\beta_{2}<\cdots$ from $D$ as follows. We let $\beta_{1}$ be the smallest element $\alpha_{\text {min }}$, and having selected $\beta_{1}$ through some $\beta_{i}$, we let $\beta_{i+1}$ be the smallest element of $D$ that is not a linear combination of $\beta_{1}, \ldots, \beta_{i}$ with nonnegative integer coefficients. We finish as soon as all of $D$ has been exhausted, and we let $\beta_{\ell}$ be the last element thus obtained.

It is easy to check that each $\beta_{i}$ is indivisible in $D$ (i.e., it is not a linear combination of other elements of $D$ with nonnegative integer coefficients). Indeed, elements of $D$ larger than $\beta_{i}$ cannot contribute to such a combination, and by the construction, $\beta_{i}$ is not a combination of smaller elements.

Now if $\ell=1$, then all dihedral angles are integer multiples of $\beta_{1}=\alpha_{\min }$. Lemma 3.2 then implies that $\pi=n \alpha_{\min }$ for some $n$. Since $\frac{\pi}{2}$ cannot be the smallest dihedral angle, we get that case (i) occurs.

If $\ell \geq 2$, then each $\beta_{i}$ is the dihedral angle of at least three edges by Lemma 3.1, and we have case (ii).

If $S$ has two distinct dihedral angles $\beta_{1} \neq \beta_{2}$, each occurring at three edges, then they are placed as in Fig. 2 left or right (up to a permutation of the vertices). We speak of the triangle-tripod configuration and the path configuration. The former is easy to deal with and we exclude it right away.

Lemma 3.4 (Excluding triangle-tripod) A tetrahedron with the triangle-tripod configuration of (at most) two dihedral angles cannot be a k-reptile (assuming, as usual, that $k$ is not a third power).

Proof Let $\beta_{1}$ be the dihedral angle at the edges of the triangle, and let $\beta_{2}$ be dihedral angle at edges of the tripod. For geometric reasons, we have $0<\beta_{1}<\frac{\pi}{2}$.

For every $\beta_{1} \in\left(0, \frac{\pi}{2}\right)$, we can construct a symmetric pyramid with an equilateral triangle as a base and with dihedral angles $\beta_{1}$ at the base (as indicated in Fig. 2). Such a pyramid has at most two distinct edge lengths, and so it cannot be a $k$-reptile by Lemma 3.1 . 
Fig. 3 The case with three dihedral angles

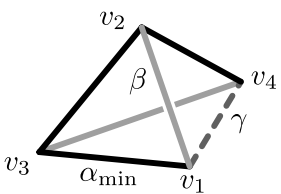

It remains to check that this pyramid is the only possible tetrahedron with the triangle-tripod configuration and with dihedral angle $\beta_{1}$ at the edges of the triangle. This can be done using Theorem 2.2, for example.

Letting $t:=\cos \beta_{1}$ and $s:=\cos \beta_{2}$, the matrix $A$ as in Theorem 2.2 is

$$
A=\left(\begin{array}{cccc}
-1 & t & t & t \\
t & -1 & s & s \\
t & s & -1 & s \\
t & s & s & -1
\end{array}\right)
$$

We have $\operatorname{det}(A)=(1+s)^{2}\left(1-2 s-3 t^{2}\right)$, and this has to be 0 according to Theorem 2.2. Hence $t$ determines $s$ uniquely, and since the dihedral angles determine a tetrahedron up to similarity (Lemma 2.4), the considered tetrahedron has to be the pyramid as claimed.

Next, we dispose with case (i) in Lemma 3.3, where all dihedral angles are integer multiples of the minimal angle.

Lemma 3.5 (Multiples of $\alpha_{\min }$ ) A tetrahedron where the minimal dihedral angle $\alpha_{\min }=\frac{\pi}{n}$ for an integer $n \geq 3$ and all other dihedral angles are integer multiples of $\alpha_{\min }$ cannot be a k-reptile.

Proof The angle $\alpha_{\min }$ occurs on at least three edges by Lemma 3.1, and thus it occurs at least twice at some vertex. Let $\beta$ be the third angle at such a vertex (possibly equal to $\alpha_{\min }$ ); we have $2 \alpha_{\min }+\beta>\pi$ (Observation 2.5).

Writing $\beta=m \alpha_{\min }=\frac{m}{n} \pi$, we thus have $2 \frac{\pi}{n}+\frac{m}{n} \pi>\pi$, which means $m>n-2$. Since $m<n$, we have $m=n-1$ and $\beta=\pi-\alpha_{\min }$. So $\beta$ is the largest dihedral angle.

Now we distinguish several cases depending on the position of the (at least three) edges with $\alpha_{\min }$.

- If they form a triangle, then all the other edges must have the angle $\beta$, and we are in the triangle-tripod case excluded by Lemma 3.4.

- If they meet at a single vertex, then $\beta=\alpha_{\min }$, and thus $\alpha_{\min }=\frac{\pi}{2}$, which is a contradiction (we know that $n \geq 3$ ).

- It remains to deal with the case where the angles $\alpha_{\min }$ occur along a path; then two edges have the angle $\beta$, and the remaining edge has some angle $\gamma$ (Fig. 3).

With $t:=\cos \alpha_{\min }, \cos \beta=\cos \left(\pi-\alpha_{\min }\right)=-t$, and $u:=\cos \gamma$, the matrix $A$ in Theorem 2.2 is

$$
A=\left(\begin{array}{cccc}
-1 & -t & t & t \\
-t & -1 & u & t \\
t & u & -1 & -t \\
t & t & -t & -1
\end{array}\right)
$$


The first and last rows add to $(t-1,0,0, t-1)$, but, since $t<1$, this contradicts Corollary 2.3.

The lemma is proved.

So now we are left with two distinct dihedral angles $\beta_{1}, \beta_{2}$ forming the path configuration. The next lemma further restricts their values.

Lemma 3.6 If $S$ has two dihedral angles $\beta_{1} \neq \beta_{2}$ forming the path configuration, then $\max \left(\beta_{1}, \beta_{2}\right)>\frac{\pi}{3}$, and one of the following cases occurs:

(i) one of $\beta_{1}, \beta_{2}$ equals $\frac{\pi}{n}$ for some natural number $n \geq 2$, or

(ii) $\beta_{1}+\beta_{2}=\pi$.

Proof By Lemma 3.2, we have $n_{1} \beta_{1}+n_{2} \beta_{2}=\pi$ for some nonnegative integers $n_{1}, n_{2}$. If one of $n_{1}, n_{2}$ is 0 , we have case (i), so we assume $n_{1}, n_{2} \geq 1$.

By Observation 2.5, the sum of the dihedral angles incident to each vertex is strictly larger than $\pi$. For the path configuration (Fig. 2 left), this yields both $\beta_{1}+2 \beta_{2}>\pi$ and $2 \beta_{1}+\beta_{2}>\pi$. This shows that the only remaining possibility is $n_{1}=n_{2}=1$, giving case (ii).

The inequality $\max \left(\beta_{1}, \beta_{2}\right)>\frac{\pi}{3}$ follows from $\beta_{1}+2 \beta_{2}>\pi$ and $2 \beta_{1}+\beta_{2}>\pi$.

As the next step, we can exclude case (ii) of the previous lemma.

Lemma 3.7 (Path configuration with $\beta_{1}+\beta_{2}=\pi$ ) There are no tetrahedra with the path configuration of two dihedral angles $\beta_{1}, \beta_{2}, \beta_{1}+\beta_{2}=\pi$.

Proof Let us set $t:=\cos \beta_{1}$; then $\cos \beta_{2}=-t$. We are going to use Corollary 2.3. The matrix $A$ is

$$
\left(\begin{array}{cccc}
-1 & t & -t & -t \\
t & -1 & t & -t \\
-t & t & -1 & t \\
-t & -t & t & -1
\end{array}\right)
$$

The sum of the second and third rows is $(0, t-1, t-1,0)$ (and $t<1$ ), which contradicts Corollary 2.3. The lemma is proved.

Excluding Tetrahedra with Rational Angles By now we have hunted the possible $k$-reptile tetrahedra down to the path configuration of two dihedral angles $\beta_{1}=\frac{\pi}{n}$ and $\beta_{2}$. By Fact 2.1, $\beta_{2}$ must be a rational multiple of $\pi$.

The plan for the rest of the proof is simple: Using Theorem 2.2, we show that, for the path configuration, $\min \left(\beta_{1}, \beta_{2}\right)$ cannot be too small, and thus it suffices to consider only a small number of possible values of $n$ and the corresponding $\beta_{1}$ 's. For each of these values of $\beta_{1}$, we can determine the possible values of $\cos \beta_{2}$, which always turn out to be quartic or quadratic irrationalities, and finally, using Theorem 2.6, we check that none of them is a value of the cosine function at a rational angle. 
To execute this plan, we write $t=\cos \beta_{1}, s=\cos \beta_{2}$, and we set up the matrix $A$ as in Theorem 2.2:

$$
A=\left(\begin{array}{cccc}
-1 & t & s & s \\
t & -1 & t & s \\
s & t & -1 & t \\
s & s & t & -1
\end{array}\right) .
$$

First, we get rid of the case $n=2$, i.e., $\beta_{1}=\frac{\pi}{2}$. Then $t=0$, and $\operatorname{det}(A)=1-$ $3 s^{2}+s^{4}$. By Theorem 2.2, $A$ has to be singular, so $s$ must satisfy $1-3 s^{2}+s^{4}=0$. There are two roots of this equation in $(-1,1)$, namely, $\phi-1$ and $1-\phi$, where we introduce the useful notation $\phi=\frac{\sqrt{5}+1}{2}$ for the golden ratio.

However, using Theorem 2.6, one can easily produce a list of all quadratic irrationalities attained by the cosine function (and such a list is provided by Jahnel [13]): $\pm \cos 72^{\circ} \approx \pm 0.309, \pm \cos 45^{\circ} \approx \pm 0.707, \pm \cos 36^{\circ} \approx \pm 0.809$, and $\pm \cos 30^{\circ} \approx$ \pm 0.866 . So $\pm(\phi-1) \approx \pm 0.618$ is not such a value.

From now on, we thus assume $n \geq 3$, and since $\max \left(\beta_{1}, \beta_{2}\right)>\frac{\pi}{3}$, we have $\beta_{1}=$ $\frac{\pi}{n} \leq \frac{\pi}{3}<\beta_{2}$. Consequently, $t \in\left[\frac{1}{2}, 1\right)$.

Next, we find that the characteristic polynomial of $A$ factors reasonably nicely, and in particular, that one of the eigenvalues is

$$
\lambda_{1}=-\phi s+\frac{t}{\phi}-1
$$

Since $A$ should be negative semidefinite, we have $\lambda_{1} \leq 0$, and thus $s \geq \frac{t}{\phi^{2}}-\frac{1}{\phi}$. Using $t \geq \frac{1}{2}$, we have $s \geq-0.43$, and thus $\beta_{2}=\arccos s \leq \arccos (-0.43)<\frac{2 \pi}{3}$. Then, using the "vertex inequality" $2 \beta_{1}+\beta_{2}>\pi$, we obtain $\beta_{1}>\frac{\pi}{6}$. Hence, we have restricted the possible values of $\beta_{1}$ to $\frac{\pi}{3}, \frac{\pi}{4}$, and $\frac{\pi}{5}$.

Assuming $\beta_{1}=\frac{\pi}{5}$, the inequality $2 \beta_{1}+\beta_{2}>\pi$ yields $\beta_{2}>\frac{3}{5} \pi$, and thus $s<$ $\cos \frac{3}{5} \pi=-\frac{1}{2 \phi}$, while $t=\cos \frac{\pi}{5}=\frac{\phi}{2}$. Then, however, we obtain $\lambda_{1}>0$, which is a contradiction excluding $\beta_{1}=\frac{\pi}{5}$.

It remains to consider $\beta_{1} \in\left\{\frac{\pi}{3}, \frac{\pi}{4}\right\}$. These cases correspond to actual geometric tetrahedra, and here we need to use the rationality of $\beta_{2}$.

The polynomial $\operatorname{det}(A)$ factors as

$$
-\left(s^{2}+t^{2}+s t+s+t-1\right)\left(s-\frac{t}{\phi^{2}}+\frac{1}{\phi}\right)\left(t-\frac{s}{\phi^{2}}+\frac{1}{\phi}\right) .
$$

For $\beta_{1}=\frac{\pi}{3}$, we get $t=\frac{1}{2}$, and it is clear that all $s$ with $\operatorname{det}(A)=0$ are quadratic irrationalities. There are two such $s$ in the interval $(-1,1)$, which are numerically approximately -0.427 and 0.151 . Clearly, they do not belong to the above list of quadratic irrationalities (if we wanted to avoid numerical approximation, we could also substitute the numbers from the list for $s$ and check that $\operatorname{det}(A) \neq 0)$.

Similarly, for $\beta_{1}=\frac{\pi}{4}$, we have $t=\frac{1}{\sqrt{2}}$. This time, the values of $s$ for which $\operatorname{det}(A)$ vanishes are quartic (or possibly quadratic) irrationalities, and numerically, there are two values in $(-1,1),-0.131$ and -0.348 . The list of all quartic irrationalities attained by $\cos \alpha$ at rational $\alpha$ 's (also given in [13]) goes as fol- 
lows: $\pm \cos 84^{\circ} \approx \pm 0.105, \pm \cos 75^{\circ} \approx \pm 0.259, \pm \cos 67 \frac{1}{2}^{\circ} \approx \pm 0.383, \pm \cos 54^{\circ} \approx$ $\pm 0.588, \pm \cos 48^{\circ} \approx \pm 0.669, \pm \cos 24^{\circ} \approx \pm 0.914, \pm \cos 22 \frac{1}{2}^{\circ} \approx \pm 0.924$, $\pm \cos 18^{\circ} \approx \pm 0.951, \pm \cos 15^{\circ} \approx \pm 0.966$, and $\pm \cos 12^{\circ} \approx \pm 0.978$. So the possible values of $s$ again do not occur there, and Theorem 1.1 is proved.

Remark Our considerations in the last part of the proof deal with a very special case of an interesting and possibly quite challenging problem: characterizing the tetrahedra with all dihedral angles rational. This problem has been considered by Smith [20], but unfortunately, his claimed reduction of the problem to Coxeter's classification of reflection groups seems to be unsubstantiated.

Acknowledgement We would like to thank Professor Miroslav Fiedler for an inspiring consultation.

\section{References}

1. Adler, M.: Tradeoffs in probabilistic packet marking for IP traceback. In: Proc. 34th Annual ACM Symposium on Theory of Computing, pp. 407-418 (2002)

2. Adler, M., Edmonds, J., Matoušek, J.: Towards asymptotic optimality in probabilistic packet marking. In: Proc. 37th Annual ACM Symposium on Theory of Computing, pp. 450-459 (2005)

3. Bandt, C.: Self-similar sets. V. Integer matrices and fractal tilings of $\mathbf{R}^{n}$. Proc. Am. Math. Soc. 112(2), 549-562 (1991)

4. Boltyanskii, V.G.: Hilbert's Third Problem. V.H. Winston \& Sons/John Wiley \& Sons, Washington/New York (1978). Translated by Richard A. Silverman and introduced by Albert B.J. Novikoff

5. Debrunner, H.E.: Tiling Euclidean $d$-space with congruent simplexes. In: Discrete Geometry and Convexity, New York, 1982. Ann. New York Acad. Sci., vol. 440, pp. 230-261. New York Acad. Sci., New York (1985)

6. Fiedler, M.: Geometry of the simplex in $E_{n}$. Čas. Pěst. Mat. 79, 297-320 (1954) (in Czech, with English and Russian summary)

7. Fiedler, M.: Matrices and graphs in Euclidean geometry. Preprint ITI Series 2001-041, Institute for Theoretical Computer Science, Charles University, Prague (2001) (in Czech)

8. Gelbrich, G.: Crystallographic reptiles. Geom. Dedic. 51(3), 235-256 (1994)

9. Gelbrich, G.: Self-affine lattice reptiles with two pieces in $\mathbf{R}^{n}$. Math. Nachr. 178, 129-134 (1996)

10. Goldberg, M.: Three infinite families of tetrahedral space-fillers. J. Comb. Theory, Ser. A 16, 348-354 (1974)

11. Hertel, E.: Self-similar simplices. Beiträge Algebra Geom. 41(2), 589-595 (2000)

12. Hill, M.J.M.: Determination of the volumes of certain species of tetrahedra without employment of the method of limits. Proc. Lond. Math. Soc. 2, 39-53 (1895-1896)

13. Jahnel, J.: When is the (co)sine of a rational angle equal to a rational number? Preprint, Georg-AugustUniversität Göttingen, http://www.uni-math.gwdg.de/jahnel/Preprints/cos.pdf (2010)

14. Kynčl, J., Safernová, Z.: On the nonexistence of $k$-reptile simplices in $R^{4}$. Manuscript in preparation (2011)

15. Matoušek, J.: Nonexistence of 2-reptile simplices. In: Discrete and Computational Geometry: Japanese Conference, JCDCG 2004. Lecture Notes in Computer Science, vol. 3742, pp. 151-160. Springer, Berlin (2005). Erratum at http://kam.mff.cuni.cz/matousek/no2r-err.pdf

16. Ngai, S.-M., Sirvent, V.F., Veerman, J.J.P., Wang, Y.: On 2-reptiles in the plane. Geom. Dedic. 82(1-3), 325-344 (2000)

17. Pak, I.: Lectures on Discrete and Polyhedral Geometry. Book in preparation, available at http://www.math.ucla.edu/pak/book.htm (2009)

18. Safernová, Z.: Perfect tilings of simplices. Bc. degree thesis. Charles University, Prague (2008) (in Czech)

19. Senechal, M.: Which tetrahedra fill space? Math. Mag. 54, 227-243 (1981)

20. Smith, W.D.: Pythagorean triples, rational angles, and space-filling simplices. Preprint (2003). Retrieved from http://www.math.temple.edu/wds/homepage/diophant.pdf in April 2010

21. Snover, S.L., Waiveris, C., Williams, J.K.: Rep-tiling for triangles. Discrete Math. 91(2), 193-200 (1991) 